\title{
Altair: Automatic image analyzer to assess retinal vessel caliber
}

\author{
Gabino Verde ${ }^{1}$, Luis García-Ortiz ${ }^{2}$, Sara Rodríguez ${ }^{1}$, José I. Recio-Rodríguez ${ }^{2}$, Juan \\ F. De Paz ${ }^{1}$, Manuel A. Gómez-Marcos ${ }^{2}$, Miguel A. Merchán and Juan M. Corchado ${ }^{1}$ \\ ${ }^{1}$ Computers and Automation Department, University of Salamanca, Salamanca, Spain \\ ${ }^{2}$ Primary care Research unit La Alamedilla. Sacyl. IBSAL. Salamanca. Spain. \\ \{gaby, lgarciao, srg, donrecio, fcofds, magomez, merchan, corchado\}@usal.es
}

\begin{abstract}
The scope of this work is to develop a technological platform specialized in assessing retinal vessel caliber and describing the relationship of the results obtained to cardiovascular risk. Population studies conducted have found retinal vessel caliber to be related to the risk of hypertension, left ventricular hypertrophy, metabolic syndrome, stroke, and coronary artery disease. The vascular system in the human retina has a unique property: it is easily observed in its natural living state in the human retina by the use of a retinal camera. Retinal circulation is an area of active research by numerous groups, and there is general experimental agreement on the analysis of the patterns of the retinal blood vessels in the normal human retina. The development of automated tools designed to improve performance and decrease interobserver variability, therefore, appears necessary.
\end{abstract}

Keywords: arteriolar-venular ratio; arterial stiffness; cardiovascular disease; AI algorithms; pattern recognition, image analysis; expert knowledge

\section{Introduction and background}

Image processing, analysis and computer vision techniques are increasing in prominence in all fields of medical science, and are especially pertinent to modern ophthalmology, as it is heavily dependent on visually oriented signs. Automatic detection of parameters from retinal images is an important problem since are associated with the risk of hypertension, left ventricular hypertrophy, metabolic syndrome, stroke, and coronary artery disease [21][22][23][24].

The vascular system in the human retina has a unique property: it is easily observed in its natural living state in the human retina by the use of a retinal camera. The retina is the only human location where blood vessels can be directly visualized non-invasively. The identification of landmark features such as the optic disc, fovea and the retinal vessels as reference co-ordinates is a prerequisite before systems can achieve more complex tasks identifying pathological entities. Reliable techniques exist for identification of these 
structures in retinal photographs. The most studied areas in this field can be classified into three [17]:

1. The location of the optic disc, that is important in retinal image analysis for vessel tracking, as a reference length for measuring distances in retinal images, and for identifying changes within the optic disc region due to disease. Techniques as analysis of intensity pixels with a high grey-scale value [14][6] or principal component analysis (PCA) [15] are used for the location of the disk. Others authors [13] use the Hough transform (a general technique for identifying the locations and orientations of certain types of shapes within a digital image [13] ) to locate the optic disc. A "fuzzy convergence" algorithm is other technique use for this goal [7].

2. The detection of the fovea, usually chosen as the position of maximum correlation between a model template and the intensity image [15].

3. The segmentation of the vasculature form retinal images, that is, the representation by segments or similar structures of the blood vessels and their connections. There are a lot of techniques to do this, the most significant are: (i) matched filters, which typically has a Gaussian or a Gaussian derivative profile [2] [10] [16] [11]; (ii) vessel tracking, whereby vessel centre locations are automatically sought over each crosssection of a vessel along the vessels longitudinal axis, having been given a starting and end point [20]; (iii) neural networks, which employ mathematical "weights" to decide the probability of input data belonging to a particular output [1]; (iv) morphological processing, that uses characteristics of the vasculature shape that are known a priori, such as it being piecewise linear and connected [7].

An understanding of the design principles of the human vascular system may have applications in the synthetic design of vascular systems in tissue and organ engineering, i.e., bioartificial organs for both liver and kidney. In the current scientific literature one can find a lot of researches devoted to these areas trying to automate the analysis of retinal images [19] [12] [3][5][7]. In this paper, after several years of studies and tests [5], we propose a novel platform image processing for the study of structural properties of vessels, arteries and veins that are observed with a red-free fundus camera in the normal human eye, and the fractal analysis of the branching trees of the vascular system. The platform, called Altair "Automatic image analyzer to assess retinal vessel caliber", employs analytical methods and AI (Artificial Intelligence) algorithms to detect retinal parameters of interest. The sequence of algorithms represents a new methodology to determine the properties of retinal veins and arteries. The platform does not require user initialization and is robust to the changes in the appearance of retinal fundus images typically encountered in clinical environments and is intended as a unified tool to join in all the methods needed to carry out the automation of all processes of measurement on the retinas. This uses the latest computer techniques both as statistical and medical. The next section introduces Altair platform. Section 3 presents the most important characteristics of the platform, showing some of the relevant techniques and results. Finally, in section 4 some conclusions are presented. 


\section{Platform Overview}

Altair facilitates the study of structural properties of vessels, arteries and veins that are observed with a red-free fundus camera in the normal human eye, and the fractal analysis of the branching trees of the vascular system. Figure 1 shows an example of images taken directly from the fundus. The retinal vessels appear in different color, with the optic disc and fovea.

There are many patterns in nature that show ramified as the retinal vessel, with open branching structures and different lengths. These objects can be described by fractal geometry. Different analytical methods and AI algorithms are used to determine the scaling properties of real objects yielding different measures of the fractal dimension, length and area of retinal veins and arteries.

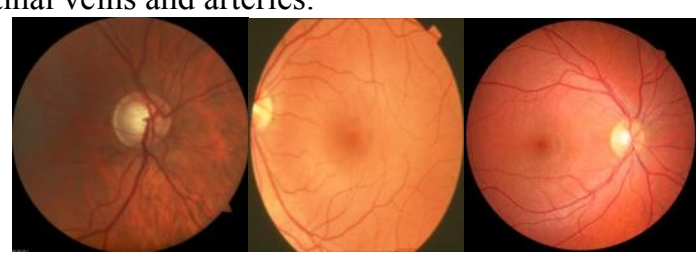

Figure 1: Retinograph usually takes three images of each eye: a) Centered papilla. b) With the disc on one side. c) With the macula and disc each to one side of center

The main objective is to relate the level of cardiovascular risk in patients with everything that it is possible to observe in the retinas. In this work we are interested in obtaining as much information as possible from the images obtained, in this case we have focused on:

- Index Artery / Vein: represents a relationship between the thickness of arteries and veins.

- Branching: branching structures: including fractal analysis of the branching trees of the vascular system. In subjects with cardiovascular diseases usually appear more branches, especially around the papilla; branching index number of times that an artery branches; branching pattern: the way in which branch arteries. Actually the manner in which branching is practically as a fingerprint of the person (each have a different shape), however many samples retinas one could observe certain normal patterns. In that case it could study the relationship of these patterns with the diseases.

- Area occupied by the veins and arteries.

- Distribution of the capillary: according to the blood distribution, the color distribution of the capillaries varies.

Moreover, our intention is to incorporate expert knowledge taken from the measures found in the retinal circulation which specify normal values of various retinal structures in healthy subjects, and to apply this information to the study of patients suffering from a number of diseases. Based on the values for area, length, position and patterns of the branching trees of the vascular system in healthy patients, we expect to determine ranges of normalcy within the population for their subsequent application to subjects affected by various diseases.

In the next section is explained the main components of the platform. The original image passes through each one of the modules (preprocessing, detection, segmentation 
and extraction of knowledge) which use different techniques and algorithms to obtain the desired image information. This sequence of steps is a methodology that is explained in the following section, also showing examples of the results obtained.

\section{Methodology and Results}

The methodology used to obtain the functionality of the platform may be divided into two phases. Firstly, a phase, called "digitization of the retina", in which the different parts of the eye image are identified. Here a data structure is created, allowing us to represent and process of the retina without requiring the original image. In this phase modules of preprocessing, detection and segmentation are included. Secondly, a phase of "measurements" in which we work with retinas that have been previously identified. In this phase is included the extraction of knowledge and the manual correction or expert knowledge if it is necessary.

In this paper we have focused in the first phase which is in charge of creating and identifying all the elements of interest of the retina. To carry out these phases, the following steps are necessary.

\section{1. Preprocessing}

The preprocessing or filtering module reduces noise, improves contrast, sharpens edges or corrects blurriness. Some of these actions can be carried out at the hardware level, which is to say with the features included with the camera. For testing, retinography was performed using a Topcon TRC NW 200 nonmydriatic retinal camera (Topcon Europe B.C., Capelle a/d Ijssel, The Netherlands), obtaining nasal and temporal images centered on the disk (Figure 1). The nasal image with the centered disk is loaded into the platform software through preprocessing module.

\subsection{Detection limits}

This module is in charge of the location of the disk and the identification of the center and edges of the retina. The goal here is to construct a data structure that identifies each part of the retina based on the matrices of colors representing the images obtained (Figure 1). In this step, image processing techniques to detect intensity based on the boundaries of the structures [7][3] have been used. The identification of the papilla is important since it serves as the starting point for the detection and identification of the different blood vessels.

In this phase boundaries are identified and the retinal papilla from a RGB image of the retina. The following values are returned: $C_{r}$ is the center of the retina, which identifies the vector with coordinates $x, y$ of the center of the retina. $C_{p}$ is the center of the disc, which identifies the vector with the coordinates $x, y$ of the center of the papilla. $R_{r}$, is the radius of the retina. $R_{p}$, is the radius of the papilla. For example, a sequence of output values in this phase is the following table and figure: 
Table 1. Sequence of output values in detection modules (pixel)

\begin{tabular}{|l|l|l|l|}
\hline$C_{r}$ & $C_{p}$ & $R_{r}$ & $R_{p}$ \\
\hline $\mathbf{1 0 1 2 , 4 4} ;$ & $\mathbf{1 0 3 5 , 9 8 ; 7 3 4 , 1 1}$ & $\mathbf{6 9 2 , 6 8}$ & $\mathbf{1 1 1 , 7 6}$ \\
$\mathbf{7 7 4 , 1 3}$ & 1104,$87 ; 562,52$ & & 108,92 \\
& 915,$38 ; 736,77$ & & 122,15 \\
& 900,$27 ; 658,74$ & & 101,95 \\
& & & \\
\hline
\end{tabular}

Figure 2: Identification result in the detection phase.

To carry out the identification of the limits, and in particular to the identification of the circumferences, it became necessary to carry out a process of image segmentation. Segmentation is the process that divides an image into regions or objects whose pixels have similar attributes. Each segmented region typically has a physical significance within the image. It is one of the most important processes in an automated vision system because allows to extract the objects from the image for subsequent description and recognition. Segmentation techniques can be grouped in three main groups: techniques based on the detection of edges or borders [13], thresholding techniques [14] and techniques based on clustering of pixels [6]. After analyzing the possibilities we chose one of the techniques of the first group that provided the best results. In this case using an optimization of the Hough transform [13]. This technique is very robust against noise and the existence of gaps in the border of the object. It is used to detect different shapes in digital images. When applying the Hough transform to an image must first obtain a binary image of the pixels that form part of the limits of the object (applying edge detection). The aim of the Hough transform is found aligned points that may exist in the image to form a desired shape. For example, to identify a line points that satisfy the equation of the line: $(\rho=x \cdot \cos \theta+\operatorname{sen} \theta$, in polar coordinate). In our case, we look for points that verify the equation of the circle: (i) in polar coordinate system: $r^{2}-2 s r \cdot \cos (\theta$ - $\alpha)+s^{2}=c^{2}$, where $(s, \alpha)$ is the center and $c$ the radius; (ii) in cartesian coordinate system: $(x-a)^{2}+(y-b)^{2}=r^{2}$, where $(a, b)$ is the center and $r$ the radius.

For the algorithm is not computationally heavy, does not check all radios, or all possible centers, only the candidate values. The candidates centers are those defined in a near portion of the retina and the radius are in approximately one sixth the radius of the retina. To measure the approximate diameter of the retina, the algorithm calculates the average color of the image column: diameter of the retina is the length that has non-zero value (black).

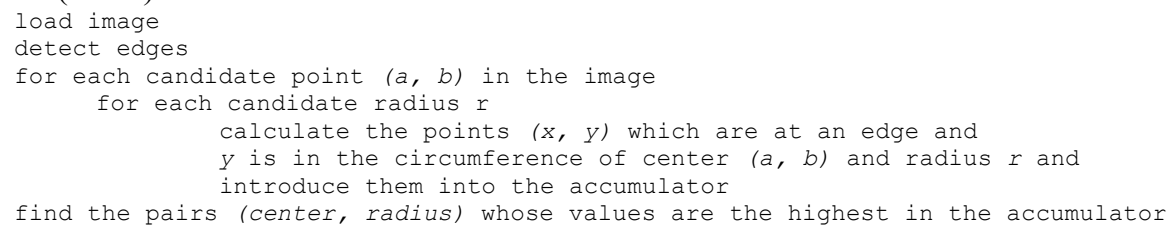

Figure 3: Pseudocode of the identification algorithm of the papilla

Having identified the papilla (Figure 3) is a necessary step because it provides a starting point for other stages of segmentation and reference point for some typical measurements. Typically the correct result is the circumference of the higher value in the 
accumulator (over $70 \%$ of cases). In almost $100 \%$ of the cases, the correct identification is between the first 3 greatest accumulator values having.

\subsection{Segmentation of the vasculature from retinal images}

The ultimate goal in this module is to identify each blood vessel as a series of points that define the path of the vessel. Each of these points will be assigned a certain thickness. Moreover, it must distinguish whether a particular blood vessel is a vein or an artery. AI algorithms responsible for identifying veins and arteries must perform a series of sweeps in search of "key points". Algorithms based on matched filters[2] [10] [16] [11], vessel tracking [19] and PCA [15], among others, are used for obtaining the proximity points between objects (veins, arteries, capillaries), the structures retinal structures or assemblies, branching patterns, etc. These algorithms work with transformations of the original image of the retina obtained of the previous step. Within this module it is necessary three steps: (i) identification of vessels; (ii) definition of the structure of vessel; (iii) cataloging of veins and arteries.

\section{3.1 Identification of vessels}

In this step the blood vessels are identified in the image by thresholding techniques. Its purpose is to remove pixels where no enters the structuring element, in this case the blood vessels. The image on the retina is blurred to keep an image similar to the background. This image is used as a threshold so that the pixels of the original image will be treated as vessels if its intensity reaches $90 \%$ of the background intensity.
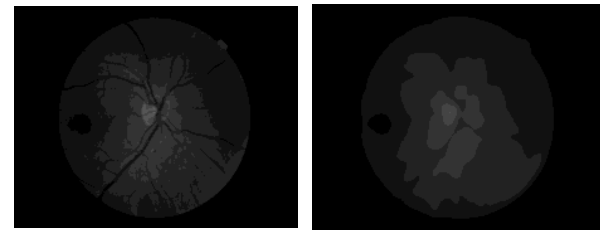

Figure 4: a) Original image in the green channel and applying a filter media 3 times with a $3 \times 3$ window to remove noise . b) Background image (to calculate it is applied on (30 times) a filter media with a $15 \times 15$ window).

The image below represents the application of these techniques in a row. The blue line represents the values of the pixels in the image; the red line, the background values; and the green line the point where there is a vessel:
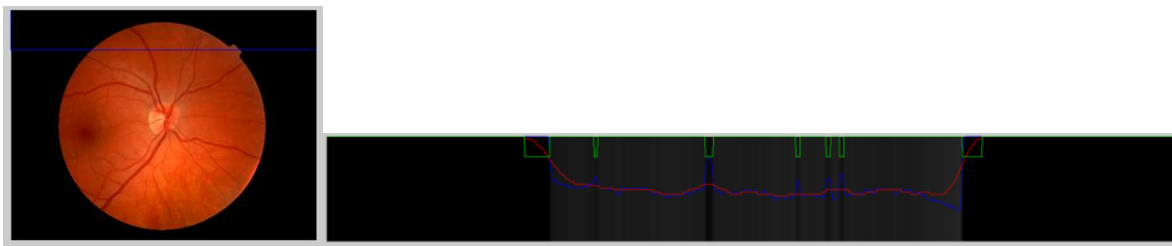

Figure 5: Thresholding techniques for the identification of vessels

In the figure, it is possible to observe that on the left there is a very small vessel artery from below. In the middle is a fat vein and right three tiny vessels. Furthermore the edge of the retina is marked as a vessel although obviously not is. To decide where there is a 
vessel and where, it applies the following algorithm (Figure 6a). Where Original $(x, y)$ is the pixel $(x, y)$ of the original image and Background $(x, y)$ is the pixel $(x, y)$ of the background image. The result is showed in the Figure $6 \mathrm{~b}$.
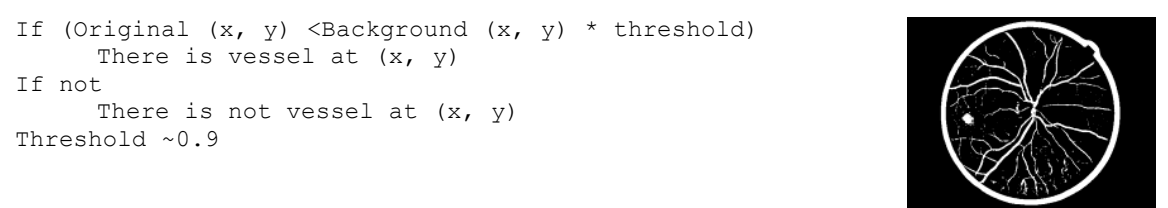

Figure 6: a) Pseudocode of the identification algorithm of the papilla. b) Image result. (Blank pixels are the vessels)

\section{3.2 Structure of vessel}

This phase defines the tree forming blood vessels. Various techniques are used in conjunction with the following steps:

Step 1: Identification of connected components

- Dilate the binary image of the vessels (horizontally and vertically) to join possible discontinuities.

- Identify the connected component of the papilla. The remainder are discarded, will mostly noise points. Labeling.

Step 2: Morphological image processing.

- Choose corona of 10 pixels thick around the disc.

- Search regions in the corona. The center of gravity of each region found is taken as the starting point of a vessel.

- Choose another corona of 10 pixels around the former so that they overlap slightly.

- Search regions in the new corona. The center of gravity of each region corresponds to a found point of a vessel skeleton. If a region of this corona shares some pixel with the previous corona, join both points (nodes) with an edge.

- Repeat the above two points to cover the entire surface of the retina.

Step 3: Resolution of conflicts such as vessel bifurcations or crossovers.

Figure 7: Pseudocode of the identification algorithm of the structure of the vessels

The following image shows the output of this phase. At the end of this stage the whole arterio-venous tree is stored in a structured way that not only allows to know if a vessel passes for a point or not, but it also is known through which passes each vessel, which is its parent, etc.
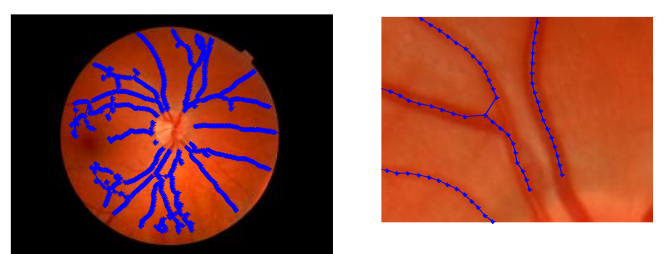

Figure 8: Structure of the vessels

\subsection{Cataloging of veins and arteries}

To detect whether a vessel is vein or artery, main branch is taken of the vessel. For every point $(x, y)$ of the branch: 

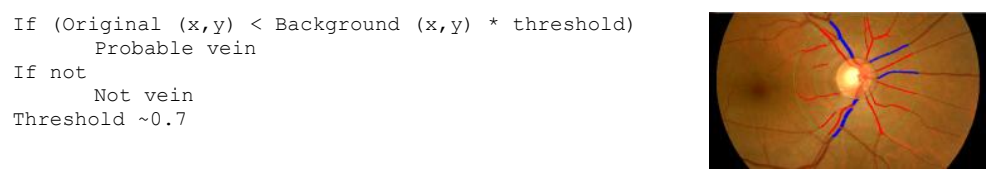

Figure 9: a) Pseudocode of the identification algorithm of veins. b) Arteries and veins detection

In general, if most of the points of the main branch of the vessel (from $60 \%$ ) are points classified as "probable vein", we conclude that this vessel is a vein, otherwise an artery. Currently, there are no publicly available databases that can be used to assess the performance of automatic detection algorithms on retinal images. In this work, we assessed the performance of our platform using retinal images acquired from Primary Care Research Unit La Alamedilla, SACYL, IBSAL, Salamanca, Spain . The images were obtained using a TopCon TRC-NW6S Non-Mydriatic Retinal Camera. Table 2 show the testing performed using 10 retinal images. No difference was found between values in terms of age, sex, cardiovascular risk factors, or drug use. The first row of values is shown in the examples retina and previous figures in this paper. The table shows: Area veins and arteries, Diameter of veins and arteries (D), AV index (AV), Veins $P\left(V_{P}\right)=$ number of veins around the papilla, Veins $A\left(V_{A}\right)=$ number of veins that cross the corona outlined with radius $=2 * R_{p}$. $R_{p}$ is the radio of the papilla, Veins $B\left(V_{B}\right)=$ number of veins that cross the corona outlined with radius $=3 * R_{p}$, same values for arteries. And the ratios leaving the region around the papilla and out of the disc (which could serve as a reference of bifurcations that has been, though not in the manner in which they branch).

Table 2. Output results for 10 retinal images

\begin{tabular}{|l|l|l|l|l|l|l|l|l|l|l|l|l|l|l|}
\hline Ret & $\begin{array}{l}\text { Area veins } \\
(\mathrm{mm} 2)\end{array}$ & $\begin{array}{l}\text { Area arteries } \\
(\mathrm{mm} 2)\end{array}$ & $\mathrm{R}_{\mathrm{p}}(\mathrm{mm})$ & $\mathrm{V}_{\mathrm{P}}$ & $\mathrm{V}_{\mathrm{A}}$ & $\mathrm{V}_{\mathrm{B}}$ & $\mathrm{A}_{\mathrm{P}}$ & $\mathrm{A}_{\mathrm{A}}$ & $\mathrm{A}_{\mathrm{B}}$ & $\begin{array}{l}\text { Thickness } \\
(\mathrm{mm})\end{array}$ & $\begin{array}{l}\text { Arteries } \\
\text { Thickness } \\
(\mathrm{mm})\end{array}$ & AV Index & Veins B/P & Veins B/P \\
\hline 1 & 2,200147433 & 1,597102803 & 1,13693 & 5 & 6 & 8 & 7 & 9 & 12 & 0,12084217 & 0,083101875 & 0,68768936 & 1,6 & 1,7142857 \\
\hline 2 & 2,004176495 & 1,995543005 & 1,04058 & 4 & 5 & 6 & 5 & 8 & 11 & 0,15342774 & 0,098325175 & 0,64085657 & 1,5 & 2,2 \\
\hline 3 & 1,637113923 & 1,377180893 & 0,973135 & 5 & 6 & 6 & 4 & 6 & 10 & 0,12165151 & 0,08752434 & 0,71946776 & 1,2 & 2,5 \\
\hline 4 & 1,698848018 & 1,463144459 & 1,05985 & 5 & 6 & 8 & 6 & 9 & 10 & 0,12091925 & 0,08029809 & 0,66406375 & 1,6 & 1,6666667 \\
\hline 5 & 2,04780811 & 1,156423484 & 1,0357625 & 6 & 6 & 10 & 6 & 6 & 8 & 0,112816215 & 0,098999625 & 0,87753011 & 1,666666667 & 1,3333333 \\
\hline 6 & 1,845338847 & 1,641012918 & 1,07912 & 5 & 5 & 7 & 7 & 8 & 11 & 0,11180454 & 0,083313845 & 0,74517408 & 1,4 \\
\hline 7 & 1,737095306 & 1,240159053 & 1,0800835 & 5 & 7 & 8 & 8 & 8 & 11 & 0,12290406 & 0,07945021 & 0,64644089 & 1,6 & 1,5714286 \\
\hline 8 & 1,612513118 & 1,261882027 & 1,0396165 & 5 & 6 & 8 & 7 & 10 & 12 & 0,12195983 & 0,08199385 & 0,6723021 & 1,6 & 1,375 \\
\hline 9 & 1,566189339 & 1,183159453 & 0,9008725 & 6 & 7 & 8 & 6 & 7 & 7 & 0,100271445 & 0,094124315 & 0,93869511 & 1,33333333 & 1,1666667 \\
\hline 10 & 1,609078289 & 1,13962067 & 0,934595 & 6 & 7 & 8 & 5 & 6 & 9 & 0,13317497 & 0,099808965 & 0,74945739 & 1,33333333 & 1,8 \\
\hline
\end{tabular}

It is possible to observe the measurement values of veins and arteries (thickness, area) are similar between different retinas (in this case not introduced any sick patient retinal image). Parameters like the veins in the papilla and AV index are the most fluctuating. 
Due to the lack of a common database and a reliable way to measure performance, it is difficult to compare our platform to those previously reported in the literature. Although some authors report algorithms and methods [19] [12] [3][5] [7] with similar performance than our platform, these results may not be comparable, since these methods are testing separately and were assessed using different databases. Since automation has been valid and verified our next step is to compare the values obtained with significant medical values in our database.

\section{4. Knowledge extraction}

This platform will show a high intra-observer and inter-observer reliability with the possibility of expert corrections if it is necessary. Results of its validity analysis must be consistent with the findings from large studies conducted with regards to both cardiovascular risk estimation and evaluation of target organ damage. The results obtained during the use of the platform will be connected and used to extract additional information by using reasoning models, such as case-based reasoning (CBR) [4][18].Taken into account the measures found in the retinal circulation which specify normal values of various retinal structures in healthy subjects, it is possible to apply this information to the study of patients suffering diseases. Moreover, because of its semiautomated nature and rapid assessment of retinal vessels, it may be helpful in clinical practice.

\section{Conclusions}

Platforms as Altair, that allows the automated diagnosis of retinal fundal images using digital image analysis offers a lot of benefits. In a research context, it offers the potential to examine a large number of images with time and cost savings and offer more objective measurements than current observer-driven techniques. Advantages in a clinical context include the potential to perform large numbers of automated screening for conditions such as the risk of hypertension, left ventricular hypertrophy, metabolic syndrome, stroke, and coronary artery disease, and hence to reduce the workload required from medical staff. As line future in this point, the next work is to analysis the significance of the measures obtained regarding their meaning in a medical context. That is, to describe the relationship of the results obtained to cardiovascular risk estimated with the Framingham or similar scale and markers of cardiovascular target organ damage. The platform is intended as a unified tool to join in all the methods needed to carry out the automation of all processes of measurement on the retinas. This uses the latest computer techniques both as statistical and medical. Thanks to the experience of the research group at the University of Salamanca (bisite.usal.es), another line of the future, which is already underway, is the migration of the platform to a cloud environment in which all services are accessible by users regardless of their location and safely and ubiquitous [8].

\section{References}

[1]. Akita, K., Kuga. H. A computer method of understanding ocular fundus images. Pattern Recogn., 16 (1982), pp. 431-443 
[2]. Chaudhuri, S., Chatterjee, S., Katz, N., Nelson, M., Goldbaum, M., 1989a. Automatic detection of the optic nerve in retinal images. In: Proceedings of the IEEE International Conference on Image Processing, vol. 1. Singapore, pp. 1-5.

[3]. Chen, B., C. Tosha, M.B. Gorin, S. Nusinowitz. Analysis of Autofluorescent retinal images and measurement of atrophic lesion growth in Stargardt disease. Experimental Eye Research, Volume 91, Issue 2, August 2010, Pages 143-152

[4]. De Paz J.F., Rodríguez S., Bajo J., Corchado J.M. CBR System for Diagnosis of Patient. Pags.: 807-812 pags. Editorial / Publisher: IEEE Computer Society Press. Proceedings of HIS 2008. ISBN: 978-0-7695-3326-1. 2009

[5]. García-Ortiz, José I. Recio-Rodríguez, Javier Parra-Sanchez, Luis J. González Elena, María C. PatinoAlonso, Cristina Agudo-Conde, Emiliano Rodríguez-Sánchez, Manuel A. Gómez-Marcos, on behalf of the Vaso-risk group- A new tool to assess retinal vessel caliber. Reliability and validity of measures and their relationship with cardiovascular risk. www.jhypertension.com. Volume 30 Number . April 2012

[6]. Goldbaum, M. Katz, N. , Nelson, M., Haff L.The discrimination of similarly colored objects in computer images of the ocular fundus. Invest. Ophthalmol. Vis. Sci., 31 (1990), pp. 617-623

[7]. Heneghan, C., J. Flynn, M. O'Keefe, M. Cahill. Characterization of changes in blood vessel and tortuosity in retinopathy of prematurity using image analysis. Med. Image Anal., 6 (2002), pp. 407-429

[8]. Heras, E., F. De la Prieta, V. Julian, S. Rodríguez, V. Botti, J. Bajo and J.M. Corchado. Agreetment technologies and their use in cloud computing environments. In: Progress in Artificial Intelligence. Volume 1. Number 4. (2012).

[9]. Hoover, A., Goldbaum, M. Locating the optic nerve in a retinal image using the fuzzy convergence of the blood vessels. IEEE Trans. Biomed. Eng., 22 (2003), pp. 951-958

[10]. Hoover, A., Kouznetsoza, V., Goldbaum, M. Locating blood vessels in retinal images by piecewise threshold probing of a matched filter response.IEEE Trans. Med. Imag., 19 (2000), pp. 203-210

[11]. Hunter, A., Lowell, J., Steel, D., Basu, A., Ryder, R., 2002. Non-linear filtering for vascular segmentation and detection of venous beading. University of Durham.

[12]. Jagoe Roger, J. Arnold, C. Blauth, P.L.C. Smith, K.M. Taylor, R. Wootton. Measurement of capillary dropout in retinal angiograms by computerised image analysis. Pattern Recognition Letters, Volume 13, Issue 2, February 1992, Pages 143-151.

[13]. Kalviainen, H., Hirvonen, P., Xu, L., Oja E. Probabilistic and non-probabilistic Hough transforms. Image Vision Comput., 13 (1995), pp. 239-252

[14]. Lee, S. ,Wang, Y., Lee E., A computer algorithm for automated detection and quantification of microaneurysms and haemorrhages in color retinal images.SPIE Conference on Image Perception and Performance, vol. 3663 (1999), pp. 61-71

[15]. Li,H., Chutatape, O. Automated feature extraction in color retinal images by a model based approach IEEE Trans. Biomed. Eng., 51 (2004), pp. 246-254

[16]. Lowell, J. A. Hunter, D. Steel, A. Basu, R. Ryder, L. Kennedy. Measurement of retinal vessel widths from fundus images based on 2-D modeling. IEEE Trans. Biomed. Eng., 23 (2004), pp. 1196-1204

[17]. Patton, Niall,Tariq M. Aslam, Thomas MacGillivray, Ian J. Deary, Baljean Dhillon, Robert H. Eikelboom, g, Kanagasingam Yogesan, Ian J. Constable (2006) Retinal image analysis: Concepts, applications and potential. Progress in Retinal and Eye Research.Elsevier. Volume 25, Issue 1, January 2006, Pages 99-127

[18]. Rodríguez S., De Paz J.F., Bajo J. and Corchado J.M. Applying CBR Sytems to Micro-Array Data Classification. Springer Velag. Advances in Soft Computing Series. Proceedings of IWPACBB 2008. ISBN: $\quad$ 978-3-540-85860-7. 2010

[19]. Sánchez, C., Hornero, R., López, M.I., Aboy, M., Poza, J., Abásolo, D. A novel automatic image processing algorithm for detection of hard exudates based on retinal image analysis. Medical Engineering \& Physics, Volume 30, Issue 3, April 2008, Pages 350-357

[20]. Tamura, S., Okamoto, Y., Yanashima, K. Zero-crossing interval correction in tracing eye-fundus blood vessels Pattern Recogn., 21 (1988), pp. 227-233

[21]. Tanabe Y, Kawasaki R, Wang JJ, Wong TY, Mitchell P, Daimon M, et al. Retinal arteriolar narrowing predicts 5-year risk of hypertension in Japanese people: the Funagata study. Microcirculation 2010; 17:94-102.

[22]. Wong TY, Duncan BB, Golden SH, Klein R, Couper DJ, Klein BE, et al. Associations between the metabolic syndrome and retinal microvascular signs: the Atherosclerosis Risk In Communities study. Invest Ophthalmol Vis Sci 2004; 45:2949-2954.

[23]. Wong TY, Klein R, Sharrett AR, Duncan BB, Couper DJ, Tielsch JM, et al. Retinal arteriolar narrowing and risk of coronary heart disease in men and women. The Atherosclerosis Risk in Communities Study. JAMA 2002; 287:1153-1159.

[24]. Yatsuya H, Folsom AR, Wong TY, Klein R, Klein BE, Sharrett AR. Retinal microvascular abnormalities and risk of lacunar stroke: Atherosclerosis Risk in Communities Study. Stroke 2010; 41:1349-1355. 